\title{
POINT OF VIEW
}

\section{Budesonide for ulcerative colitis}

\author{
I. Marín-Jiménez and A. S. Peña ${ }^{1}$ \\ Department of Gastroenterology. Hospital General Universitario Gregorio Marañón. Madrid, Spain \\ ${ }^{\prime}$ Departments of Gastroenterology and Pathology. VU University Medical Center. Amsterdam, the Netherlands
}

\begin{abstract}
In this review, we examined studies published on oral and topical formulations of budesonide (Entocort ${ }^{\circledR}$ and Budenofalk ${ }^{\circledR}$, in Spain: Entocord ${ }^{\circledR}$ and Intestifalk ${ }^{\circledR}$ ) for the treatment of ulcerative colitis. This glycocorticosteroid has a potent local action and an important first-pass liver metabolism. It has proven successful over the last years as a controlled-release formulation. It obtained results similar to prednisolone, without the latter's significant suppression of plasma cortisol. Many publications exist on the effects of oral budesonide for the treatment of Crohn's disease (CD). These have led to the registration of this drug for the treatment of $\mathrm{CD}$. Studies on oral formulations of budesonide for the treatment of ulcerative colitis (UC) are scarce. After reviewing published evidence, we suggest the conduction of controlled trials for the treatment of UC to obtain evidence-based efficacy and safety results in order to benefit patients with this form of inflammatory bowel disease (IBD).
\end{abstract}

Key words: Budesonide. Ulcerative colitis. Inflammatory bowel disease. Treatment.

Marín-Jiménez I, Peña AS. Budesonide for ulcerative colitis. Rev Esp Enferm Dig 2006; 98: 362-373.

\section{INTRODUCTION}

The notorious frequent adverse effects associated with the use of classical glycocorticosteroids (GCSs) prompted the development of a new group of drugs with equiva-

Recibido: 04-11-05.

Aceptado: 08-11-05.

Correspondencia: A. S. Peña. Department of Gastroenterology. Laboratory of Immunogenetics. Department of Pathology. VU University Medical Center. P.O. Box 7057. 1007 MB Amsterdam. The Netherlands. Fax: +31 20444 8418. Fax direct: + 3120444 4737. e-mail: as.pena@vumc.nl lent efficacy and a safer profile. The pharmacological development of novel GCSs has been more difficult in UC than in other diseases because of variations in colonic $\mathrm{pH}$, transit time, and the effects of bacterial metabolism. Friend (1) has evaluated prednisolone metasulfobenzoate, fluticasone propionate, tixocortol pivalate, beclomethasone dipropionate, and budesonide. Budesonide, with its high topical activity and high rate of first-pass metabolism in oral controlled-release formulations, can reach different bowel compartments. This has led to the qualification of "a model of targeted therapy" (2). Two commercially available enteric-coated $\mathrm{pH}$-dependent release oral formulations have been marketed, Entocord ${ }^{\circledR}$ EC and Budenofalk ${ }^{\circledast}$ (in Spain Entocord ${ }^{\circledR}$ and Intestifalk ${ }^{\circledast}$ ). The latter will be available in Spain in 2006. Budesonide gives an overall treatment result approaching that of prednisolone but without the suppression of plasma cortisol levels (3). Many studies have been published on the effects of oral budesonide in Crohn's disease (CD) (4-6), but there is little evidence on the efficacy of oral budesonide in UC. The importance of fewer side effects in the case of GCSs will undoubtedly help improve patient compliance regarding treatment (7). In a recent study of the incidence of UC in northern Spain, disease extension was limited to proctitis in $11 \%$ of patients, left-side colitis in 53\%, and extensive colitis in $36 \%$ of patients (8). This suggests that both topical and oral therapy with GCSs need optimization.

The efficacy and fewer side effects of enema/foam preparations of budesonide for the treatment of distal disease in UC are encouraging. An excellent review of budesonide published in 1999 by Gomollón et al. has analyzed the value of this drug in the treatment of $\mathrm{CD}$ and UC (9). Since then, new papers with novel data exist, although some uncertainties remain. In this revision, we wish to reassess data published in abstracts from different national and international congresses, as well as the bibliography provided by pharmaceutical companies. We 
used Medline's systematic search tools for the use of topical GCSs in CD and UC, and we screened for studies published on the presently available enteric-coated $\mathrm{pH}$ dependent release oral formulations, as well as on the topical preparations for distal IBD.

We divided information into the following sections:

1. Budesonide Pharmacology.

2. Studies of Budesonide for Ulcerative Colitis:

-Topical budesonide: pharmacokinetic studies.

- Topical budesonide compared with placebo.

-Topical budesonide compared with topical corticosteroids.

-Topical budesonide compared with topical aminosalicylates.

-Topical budesonide compared with oral metronidazole.

-Oral budesonide.

3. Future perspectives.

\section{BUDESONIDE PHARMACOLOGY}

In contrast to other steroids such as hydrocortisone, prednisolone and dexamethasone (10), budesonide is a non-halogenated synthetic corticosteroid with the highest affinity for the glycocorticoid receptor. Budesonide is a 1: 1 mixture of two epimers (22R and 22S). Both epimers are rapidly eliminated with a terminal half-life of 2.7 \pm 0.6 hours (11). Budesonide is extensively metabolized by hydroxylation, while the cytochrome $\mathrm{P} 450$ isoenzyme CYP3A4, expressed in high amounts in hepatocytes and epithelial cells of the intestinal wall, is the main responsible isoenzyme for its rapid elimination (12).

Budesonide circulates in the plasma mainly bound to proteins $(88 \%)$. With a dosage range of $3-15 \mathrm{mg} / \mathrm{day}$, it shows a linear pharmacokinetic behavior $(13,14)$. Due to the high clearance of budesonide, which approaches the clearance of liver blood flow, a low oral bioavailability has to be expected. After oral administration and absorption, budesonide undergoes a $90 \%$ first-pass hepatic metabolism (15). It is transformed into 6-beta-hydroxibudesonide and 16 alpha-hydroxiprednisolone; both of these contain less than $1 \%$ of the parent compound. This explains the mere $10 \%$ of oral bio-availability and the low systemic action of budesonide. When administered as an enema to humans, budesonide reaches the splenic flexure (16). Its bioavailability then averages $15 \%$ in patients with UC. Some animal experiments (17) have revealed that budesonide has a longer retention in the colonic mucosa versus systemic corticosteroids; 20 minutes and 4 hours after perfusion of the rat colon, higher concentrations of budesonide were detected when compared to prednisolone.

There are three different forms of oral controlled-released preparations of budesonide (18): controlled ilealrelease capsules, a $\mathrm{pH}$-modified release formulation, and a budesonide prodrug (budesonide-beta-D-glucuronide).
The latter preparation is not available yet (18). The controlled ileal-release formulation (Entocort ${ }^{\circledR}$ EC) is composed of a hard gelatin capsule with acid-resistant pellets covered with Eudragit L 100-55; it has a delayed release at $\mathrm{pH}>5.5$. The $\mathrm{pH}$-modified release formulation (Budenofalk ${ }^{\circledR}$ ) is also composed of a gelatin capsule and acidresistant pellets; however the pellets are covered with Eudragit L, S, LS, and RS, and have a delayed release at $\mathrm{pH}>6.4$. Budesonide-beta-D-glucuronide is an oral prodrug targeted to deliver budesonide specifically to the colon, since this prodrug is not absorbed in the small intestine. Budesonide-beta-D-glucuronide is hydrolyzed by colonic bacterial and mucosal beta-glucuronidase in order to release free budesonide into the colon (19). Hydrolysis rates of budesonide-beta-D-glucuronide in human fecal samples from patients with UC and normal volunteers are similar (20), but it is not clear whether a $\mathrm{pH}$ reduction in the colon of IBD patients may inhibit the bacterial hydrolysis of this prodrug.

\section{STUDIES OF BUDESONIDE IN ULCERATIVE COLITIS}

\section{Topical budesonide: pharmacokinetic studies}

In table I we summarized a compilation of four studies in which different pharmacokinetic aspects of budesonide in UC patients were evaluated (16,21-23). The first study (21) showed that budesonide does not accumulate in the human body after 4 weeks of treatment; also, mean plasma cortisol values did not change during this period of time. The second study (16) showed that a low viscosity formulation of budesonide had an improved capacity to reach the more proximal parts of the colon, reaching the splenic flexure in 15 minutes. In the third study (22), a dose of $2 \mathrm{mg} /$ day showed the same efficacy as the $4 \mathrm{mg} /$ day dosage, but with less plasma cortisol suppression. This third study also demonstrated that budesonide enemas given twice weekly appear to be sufficient to maintain remission and prevent relapses in patients with quiescent disease during some months after suppressing active disease. The fourth, recently published study (23) showed that budesonide foam $(20 \mathrm{ml})$ reaches the sigmoid colon after rectal application. Noteworthy is the fact that patients preferred this foam to enemas.

\section{Topical budesonide compared with placebo}

In the first of the two studies (24), shown in table II, budesonide is significantly more effective than placebo to achieve endoscopical, histological and clinical improvement in UC patients without causing a decrease in plasma cortisol levels. The second study (25), apart from comparing budesonide with placebo also evaluated three different enema dosages $-0.5,2$ and $8 \mathrm{mg}$. This study proved that 
Table I. Pharmacokinetic studies of topical budesonide

\begin{tabular}{|c|c|c|c|c|c|c|}
\hline $\begin{array}{l}\text { Year of } \\
\text { publication }\end{array}$ & $\begin{array}{l}\text { Author } \\
\text { reference }\end{array}$ & $n$ & $\begin{array}{l}\text { Ulcerative colitis } \\
\text { characteristics }\end{array}$ & $\begin{array}{l}\text { Medication } \\
\text { (dose) }\end{array}$ & $\begin{array}{l}\text { Time } \\
\text { Evaluation parameters }\end{array}$ & $\begin{array}{l}\text { Results/conclusions } \\
\text { Cortisol depression }\end{array}$ \\
\hline 1993 & $\begin{array}{l}\text { Danielsson } \\
\text { et al. (21) }\end{array}$ & 28 & $\begin{array}{l}\text { Distal active UC } \\
\text { and proctitis }\end{array}$ & $\begin{array}{l}\text { Budesonide } \\
\text { ENE }(2 \mathrm{mg})\end{array}$ & $\begin{array}{l}4 \text { weeks } \\
\text { Pharmacokinetic } \\
\text { assessment; } \\
\mathrm{E}+\mathrm{H}\end{array}$ & $\begin{array}{l}\text { Improvement of } \mathrm{E}+\mathrm{H} \\
\text { Budesonide does not accumulate } \\
\text { Mean plasma cortisol values did not change du- } \\
\text { ring treatment }\end{array}$ \\
\hline 1994 & $\begin{array}{l}\text { Nyman-Pantelidis } \\
\text { et al. (16) }\end{array}$ & 5 & $\begin{array}{l}\text { Distal active UC } \\
\text { and proctitis }\end{array}$ & $\begin{array}{l}\text { Budesonide ENE (two } \\
\text { different formulations: } \\
\text { low and high viscosity) }\end{array}$ & $\begin{array}{l}\text { Area of spread of } \\
\text { enema }\end{array}$ & $\begin{array}{l}\text { Low viscosity ENE gets to splenic flexure } \\
\text { in } 15 \text { minutes; high viscosity ENE get } \\
\text { less far and spending much more time }\end{array}$ \\
\hline 2002 & Lindgren et al. (22) & 149 & Distal active UC & $\begin{array}{l}\text { Budesonide ENE } \\
\text {-acute phase: } 2 \mathrm{mg} \\
\text { (q.d. or ti..d) until } 8 \\
\text { weeks/remission } \\
\text {-Maintenance: } 2 \mathrm{mg} / \text { twice } \\
\text { weekly until } 24 \text { week } \\
\text { or relapse }\end{array}$ & $\begin{array}{l}\text { Acute phase: } \\
8 \text { weeks } \\
\text { Maintenance: } \\
24 \text { weeks }\end{array}$ & $\begin{array}{l}\text { Remission rates at week } 4 \text { and } 8 \text { the same } \\
\text { in } 2 \text { and } 4 \text { mg groups } \\
4 \text { mg group much adrenal alteration } \\
\text { Budesonide for maintenance at this } \\
\text { intervals is not effective to prevent relapse }\end{array}$ \\
\hline 2005 & Brunner et al. (23) & 12 & $\begin{array}{l}\text { Proctosigmoiditis and } \\
\text { left sided colitis }\end{array}$ & $\begin{array}{l}\text { Budesonide FO } \\
\text { (2 mg/20 mL) }\end{array}$ & $\begin{array}{l}\text { - } \\
\text { Area of spread of } \\
\text { the drug }\end{array}$ & $\begin{array}{l}\text { Foam gets to sigma in all patients } \\
\text { Drug is detected } 4 \text { hours after instillation }\end{array}$ \\
\hline
\end{tabular}

VN: number of patients; E: endoscopic evaluation; H: histologic evaluation; Clin: clinical evaluation. ENE: enema. FO: foam.

Table II. Studies comparing topical budesonide with placebo

\begin{tabular}{|c|c|c|c|c|c|c|}
\hline $\begin{array}{l}\text { Year of } \\
\text { publication }\end{array}$ & $\begin{array}{l}\text { Author } \\
\text { reference }\end{array}$ & $n$ & $\begin{array}{l}\text { Ulcerative colitis } \\
\text { characteristics }\end{array}$ & $\begin{array}{l}\text { Medication } \\
\text { (dose) }\end{array}$ & $\begin{array}{l}\text { Time } \\
\text { Evaluation parameters }\end{array}$ & $\begin{array}{l}\text { Results/conclusions } \\
\text { Cortisol depression }\end{array}$ \\
\hline 1992 & Danielsson et al. (24) & 41 & $\begin{array}{l}\text { Distal active UC } \\
\text { and proctitis }\end{array}$ & $\begin{array}{l}\text { Budesonide ENE } \\
(2 \mathrm{mg} / 100 \mathrm{ml}) \mathrm{vs} . \\
\text { placebo ENE }\end{array}$ & $\begin{array}{l}4 \text { weeks } \\
\mathrm{E}+\mathrm{H}+\mathrm{Clin}\end{array}$ & $\begin{array}{l}\text { Budesonide more effective than placebo. } \\
\text { Budesonide not decrease cortisol plasma levels }\end{array}$ \\
\hline 1998 & Hanauer et al. (25) & 233 & $\begin{array}{l}\text { Distal active UC } \\
\text { and proctitis }\end{array}$ & $\begin{array}{l}\text { Budesonide ENE } \\
(0.5,2,8 \mathrm{mg} / 100 \mathrm{ml}) \text { vs. } \\
\text { placebo ENE }\end{array}$ & $\begin{array}{l}6 \text { weeks } \\
\mathrm{E}+\mathrm{H}+\mathrm{Clin}\end{array}$ & $\begin{array}{l}\text { Budesonide (dose: } 2 \text { and } 8 \mathrm{mg} \text { ) better than } \\
\text { placebo in } \mathrm{E}, \mathrm{H} \text { and Clin. } 90 \% \text { patients on } \\
\text { budesonide maintain normal plasma cortisol } \\
\text { levels }\end{array}$ \\
\hline
\end{tabular}

$\mathrm{N}$ : number of patients; E: endoscopic evaluation; H: histologic evaluation; Clin: clinical evaluation. ENE: enema. FO: foam.

budesonide is significantly superior to placebo in UC patients with distal active UC and proctitis. The 2-mg dose enema was recommended, as this proved to be the minimum dose to show a significant effect when compared to placebo. At week 6, a remission rate of only $19 \%$ was reported. This low rate of remission is the result of strict criteria to define "remission" in this study.

\section{Topical budesonide compared with topical corticosteroids}

Table III shows the results of budesonide for UC patients compared with the results obtained with classic corticosteroids. In two $(26,27)$ of the nine studies summarized in this table, foam was used as a vehicle for the drug. In almost all nine studies, budesonide showed a similar efficacy as compared to classic topical steroids, although with a better safety profile. Budesonide did not decrease plasma cortisol levels. When budesonide foam was compared with betamethasone enema (27), no significant differences in terms of quality of life were observed; however, betamethasone reduced plasma cortisol levels. Two metaanalysis which evaluated the efficacy of rectal budesonide versus classic corticosteroids for the treatment of distal ulcerative colitis $(28,29)$ concluded that no significant differences exist in efficacy between budesonide and classic topical corticosteroids, and that budesonide induces less endogenous cortisol suppression.

\section{Topical budesonide compared with topical aminosalicylates}

In the three studies shown in table IV, budesonide enema and foam were compared with topical 5-ASA 
Table III. Studies comparing topical budesonide with topical corticosteroids

\begin{tabular}{|c|c|c|c|c|c|c|}
\hline $\begin{array}{l}\text { Year of } \\
\text { publication }\end{array}$ & $\begin{array}{l}\text { Author } \\
\text { reference }\end{array}$ & $n$ & $\begin{array}{l}\text { Ulcerative colitis } \\
\text { characteristics }\end{array}$ & $\begin{array}{l}\text { Medication } \\
\text { (dose) }\end{array}$ & $\begin{array}{l}\text { Time } \\
\text { Evaluation parameters }\end{array}$ & $\begin{array}{l}\text { Results/conclusions } \\
\text { Cortisol depression }\end{array}$ \\
\hline 1987 & $\begin{array}{l}\text { Danielsson } \\
\text { et al. (30) }\end{array}$ & 64 & Distal active UC & $\begin{array}{l}\text { Budesonide ENE } \\
(2 \mathrm{mg} / 100 \mathrm{ml}) \text { vs. } \\
\text { prednisolone ENE } \\
(31.25 \mathrm{mg} / 100 \mathrm{ml})\end{array}$ & $\begin{array}{l}4 \text { weeks } \\
\mathrm{E}+\mathrm{H}+\mathrm{Clin}\end{array}$ & $\begin{array}{l}\text { Budesonide > prednisolone in } \mathrm{E}, \mathrm{H} \text { and } \mathrm{Clin} \\
\text { evaluations } \\
\text { Prednisolone reduces cortisol, but not budesonide }\end{array}$ \\
\hline 1991 & $\begin{array}{l}\text { Danish Budesonide } \\
\text { Study Group (31) }\end{array}$ & 146 & Distal active UC & $\begin{array}{l}\text { Budesonide ENE } \\
(1,2 \text { or } 4 \mathrm{mg} / 100 \mathrm{ml}) \\
\text { vs. prednisolone ENE } \\
(25 \mathrm{mg} / 100 \mathrm{ml})\end{array}$ & $\begin{array}{l}2 \text { weeks } \\
\mathrm{E}+\mathrm{H}+\mathrm{Clin}\end{array}$ & $\begin{array}{l}\text { All treatments improved } \mathrm{E}+\mathrm{Clin} \text { (less the } 1 \mathrm{mg} \\
\text { budesonide group). Prednisolone reduces cortisol, } \\
\text { but not budesonide }\end{array}$ \\
\hline 1994 & Bianchi Porro et al. (32) & 88 & Distal active UC & $\begin{array}{l}\text { Budesonide ENE } \\
(2 \mathrm{mg} / 100 \mathrm{ml}) \mathrm{vs} \text {. } \\
\text { methylprednisolone } \\
\text { ENE }(20 \mathrm{mg} / 100 \mathrm{ml})\end{array}$ & $\begin{array}{l}4 \text { weeks } \\
\mathrm{E}+\mathrm{H}+\mathrm{Clin}\end{array}$ & $\begin{array}{l}\text { All treatments improved } \mathrm{E}, \mathrm{H} \text {, and Clin, without } \\
\text { significant differences between them. Prednisolone } \\
\text { reduces cortisol, but not budesonide }\end{array}$ \\
\hline 1994 & Ostergaard et al. (33) & 26 & Distal active UC & $\begin{array}{l}\text { Budesonide ENE } \\
(2 \mathrm{mg} / 100 \mathrm{ml}) \text { vs. } \\
\text { prednisolone ENE } \\
(25 \mathrm{mg} / 100 \mathrm{ml})\end{array}$ & $\begin{array}{l}8 \text { weeks } \\
\text { Adrenal gland } \\
\text { suppression }\end{array}$ & Prednisolone reduces cortisol, but not budesonide \\
\hline 1994 & Lofberg et al. (34) & 100 & Distal active UC & $\begin{array}{l}\text { Budesonide ENE } \\
(2,3 \mathrm{mg} / 115 \mathrm{ml}) \mathrm{vs} \text {. } \\
\text { prednisolone ENE } \\
(31.25 \mathrm{mg} / 125 \mathrm{ml})\end{array}$ & $\begin{array}{l}8 \text { weeks } \\
\mathrm{E}+\mathrm{H}+\mathrm{Clin}\end{array}$ & $\begin{array}{l}\text { All treatments improved } \mathrm{E}, \mathrm{H} \text {, and Clin, without } \\
\text { significant differences between them. Prednisolone } \\
\text { reduces cortisol, but not budesonide }\end{array}$ \\
\hline 1994 & Tarpila et al. (35) & 72 & Proctitis & $\begin{array}{l}\text { Budesonide ENE } \\
(2 \mathrm{mg} / 100 \mathrm{ml}) \text { vs. } \\
\text { hydrocortisone acetate } \\
\text { FO }(125 \mathrm{mg} / 125 \mathrm{ml})\end{array}$ & $\begin{array}{l}4 \text { weeks } \\
\mathrm{E}+\mathrm{H}+\mathrm{Clin}\end{array}$ & $\begin{array}{l}\text { All treatments improved } \mathrm{E}, \mathrm{H} \text {, and Clin, without } \\
\text { significant differences between them. Prednisolone } \\
\text { reduces cortisol, but not budesonide }\end{array}$ \\
\hline 1995 & Bayless et al.* (36) & 184 & Distal active UC & $\begin{array}{l}\text { Budesonide ENE } \\
(2 \mathrm{mg}) \text { vs. hydrocortisone } \\
\text { ENE }(100 \mathrm{mg}) \text { vs. } \\
\text { placebo }\end{array}$ & $\begin{array}{l}6 \text { weeks } \\
\mathrm{E}+\mathrm{H}+\mathrm{Clin}\end{array}$ & $\begin{array}{l}\text { E: budesonide similar to hydrocortisone (both better } \\
\quad \text { than placebo) } \\
\mathrm{H} \text { and Clin: non significant differences between, } \\
\text { budesonide prednisolone and placebo. Prednisolone } \\
\text { reduces cortisol significantly more than budesonide }\end{array}$ \\
\hline 2003 & Bar-Meir et al. (26) & 251 & Proctosigmoiditis & $\begin{array}{l}\text { Budesonide FO }(2 \mathrm{mg}) \\
\text { vs. hydrocortisone } \\
\text { acetate FO }(100 \mathrm{mg})\end{array}$ & $\begin{array}{l}8 \text { weeks } \\
\mathrm{E}+\mathrm{H}+\mathrm{Clin}\end{array}$ & $\begin{array}{l}\text { All treatments improved } \mathrm{E}, \mathrm{H} \text {, and } \mathrm{Clin} \text {, without } \\
\text { significant differences between them. Prednisolone } \\
\text { reduces cortisol, but not budesonide }\end{array}$ \\
\hline 2004 & Hammond et al. (27) & 38 & Distal active UC & $\begin{array}{l}\text { Budesonide FO } \\
(2 \mathrm{mg} / 50 \mathrm{ml}) \text { vs. beta- } \\
\text { methasone ENE } \\
(5 \mathrm{mg} / 100 \mathrm{ml})\end{array}$ & $\begin{array}{l}4 \text { weeks } \\
\mathrm{QOL}+\mathrm{E}+\mathrm{H}+\text { Clin }\end{array}$ & $\begin{array}{l}\text { Similar efficacy and QOL with budesonide and } \\
\text { bethametasone. Bethametasone reduces cortisol, } \\
\text { but not budesonide }\end{array}$ \\
\hline
\end{tabular}

$\mathrm{N}$ : number of patients; E: endoscopic evaluation; H: histologic evaluation; Clin: clinical evaluation. ENE: enemas. FO: foam. QOL: quality of life. * Just published in abstract form.

(37-39). These studies demonstrated similar results in terms of efficacy with an excellent safety profile.

\section{Topical budesonide compared with oral metronidazole}

Only one study for the treatment of active pouchitis (40) reported the use of topical budesonide (Entocort ${ }^{\circledast}$ enema) compared with oral metronidazole. Budesonide was as efficacious as metronidazole, and showed a better ad- verse effects profile. Although more clinical trials are needed on this subgroup of patients, budesonide could be a good alternative to the existing therapies.

\section{Oral budesonide}

Only three clinical studies have been published using oral budesonide in patients suffering from UC (Table V). In the first study (3), oral budesonide (Entocort ${ }^{\circledast}, 10$ $\mathrm{mg} /$ day) showed a similar efficacy when compared to 
Table IV. Studies comparing topical budesonide with topical aminosalicylates

\begin{tabular}{|c|c|c|c|c|c|c|}
\hline $\begin{array}{l}\text { Year of } \\
\text { publication }\end{array}$ & $\begin{array}{l}\text { Author } \\
\text { reference }\end{array}$ & $n$ & $\begin{array}{l}\text { Ulcerative colitis } \\
\text { characteristics }\end{array}$ & $\begin{array}{l}\text { Medication } \\
\text { (dose) }\end{array}$ & $\begin{array}{l}\text { Time } \\
\text { Evaluation parameters }\end{array}$ & $\begin{array}{l}\text { Results/conclusions } \\
\text { Cortisol depression }\end{array}$ \\
\hline 1991 & Lamers et al. * (39) & 62 & $\begin{array}{l}\text { Proctosigmoiditis } \\
\text { and proctitis }\end{array}$ & $\begin{array}{l}\text { Budesonide ENE } \\
(2 \mathrm{mg} / 100 \mathrm{ml}) \text { vs. 5-ASA } \\
\text { ENE }(4 \mathrm{~g} / 60 \mathrm{ml})\end{array}$ & $\begin{array}{l}4 \text { weeks } \\
\mathrm{E}+\mathrm{H}+\mathrm{Clin}\end{array}$ & $\begin{array}{l}\text { Similar efficacy in } \mathrm{E}+\mathrm{H}+\mathrm{Clin} \\
\text { No adverse effects in both groups }\end{array}$ \\
\hline 1995 & Leman et al. (37) & 97 & $\begin{array}{l}\text { Distal active UC } \\
\text { and proctosigmoiditis }\end{array}$ & $\begin{array}{l}\text { Budesonide ENE } \\
(2 \mathrm{mg} / 100 \mathrm{ml}) \text { vs. 5-ASA } \\
\text { ENE (mesalazine } \\
1 \mathrm{~g} / 100 \mathrm{ml})\end{array}$ & $\begin{array}{l}4 \text { weeks } \\
\mathrm{E}+\mathrm{H}+\mathrm{Clin}\end{array}$ & $\begin{array}{l}\text { Similar efficacy in } \mathrm{E}+\mathrm{H}+\mathrm{Clin} \\
\text { No adverse effects in both groups }\end{array}$ \\
\hline 2000 & Rufle et al. (38) & 33 & $\begin{array}{l}\text { Distal active UC } \\
\text { proctosigmoiditis } \\
\text { and proctitis }\end{array}$ & $\begin{array}{l}\text { Budesonide FO } \\
(1 \mathrm{mg} / 50 \mathrm{ml})(\mathrm{bid}) \mathrm{vs} \text {. } \\
\text { mesalazine ENE } \\
(4 \mathrm{~g} / 60 \mathrm{ml} \mathrm{o.d.)}\end{array}$ & $\begin{array}{l}6 \text { weeks } \\
\mathrm{E}+\mathrm{H}+\mathrm{Clin}\end{array}$ & $\begin{array}{l}\text { Similar efficacy in } \mathrm{E}+\mathrm{H}+\mathrm{Clin} \\
\text { No influence of both treatments in cortisol } \\
\text { plasma levels }\end{array}$ \\
\hline
\end{tabular}

$\mathrm{N}$ : number of patients; E: endoscopic evaluation; H: histologic evaluation; Clin: clinical evaluation. ENE: enemas. FO: foam. *Just published in abstract form.

Table V. Studies with oral budesonide

\begin{tabular}{|c|c|c|c|c|c|c|c|}
\hline Treatment compared & $\begin{array}{l}\text { Year of } \\
\text { publication }\end{array}$ & Author & $n$ & $\begin{array}{l}\text { Ulcerative colitis } \\
\text { characteristics }\end{array}$ & Medication (dose) & $\begin{array}{l}\text { Time } \\
\text { Evaluation parameters }\end{array}$ & $\begin{array}{l}\text { Results /conclusions } \\
\text { Cortisol depression }\end{array}$ \\
\hline $\begin{array}{l}\text { Oral budesonide } \\
\text { compared to } \\
\text { predisolone }\end{array}$ & 1996 & Lofberg et al. (3) & 72 & $\begin{array}{l}\text { Active extensive and } \\
\text { left sided UC }\end{array}$ & $\begin{array}{l}\text { Budesonide (10 mg) } \\
\text { vs. prednisolone ( } 40 \mathrm{mg} \text { ) }\end{array}$ & $\begin{array}{l}9 \text { weeks } \\
\text { E and plasma } \\
\text { cortisol levels }\end{array}$ & $\begin{array}{l}\text { Same E results } \\
\text { Prednisolone supresses cortisol, } \\
\text { but not budesonide }\end{array}$ \\
\hline Oral budesonide alone & 1997 & Keller et al. (41) & 14 & $\begin{array}{l}\text { Steroid dependent UC } \\
\text { (7 pancolitis, } 3 \\
\text { extensive colitis, } \\
3 \text { left sided colitis } \\
\text { and } 1 \text { proctitis) }\end{array}$ & Budesonide $3 \mathrm{mg}$ t.d.s. & $\begin{array}{l}6 \text { months } \\
\text { Clin. and reduction of } \\
\text { systemic steroid dose }\end{array}$ & $\begin{array}{l}11 \text { out of } 14 \text { Clin. improvement and } \\
\text { ended systemic steroid treatment }\end{array}$ \\
\hline Oral budesonide alone & 2004 & Kolkman et al. (42) & 15 & Distal active UC & $\begin{array}{l}\text { Budesonide } 9 \mathrm{mg} \mathrm{o.d} \text {. } \\
\text { vs. budesonide } 3 \mathrm{mg} \text { ti.i.d }\end{array}$ & $\begin{array}{l}8 \text { weeks } \\
\text { Pharmacokinetics, } \\
\text { pharmacodynamics, } \\
\text { safety and efficacy }\end{array}$ & $\begin{array}{l}\text { Better results in } 9 \mathrm{mg} \text { o.d group. } \\
\text { Budesonide reaches the distal part of } \\
\text { colon and the rectum }\end{array}$ \\
\hline
\end{tabular}

N: number of patients; E: endoscopic evaluation; H: histologic evaluation; Clin: clinical evaluation. ENE: enemas. FO: foam.

prednisolone (40 mg/day) in active extensive and leftsided UC. Budesonide did not modify plasma cortisol levels. The second study (41) reported the use of oral budesonide (Budenofalk ${ }^{\circledast}$ ) for steroid-dependent UC patients with disease extension from pancolitis to proctitis. Eleven out of fourteen patients achieved clinical improvement. Budesonide allowed ending the steroid treatment. The third study, in patients with distal active UC, oral budesonide (Budenofalk ${ }^{\circledR}$ ) showed encouraging clinical results (42). This study, particularly designed to study the pharmacokinetics and pharmacodynamics of Budenofalk ${ }^{\circledast}$, found significant levels of budesonide in the distal colon and rectum. This suggests that this formulation could be of value in the treatment of distal disease.

\section{FUTURE PERSPECTIVES}

The data reviewed have shown that topical budesonide is a good alternative for topical 5-ASA, the pre- sent therapy of choice, in the treatment of distal active UC. Budesonide is as effective as topical 5-ASA, with also a good safety profile. Budesonide does not decrease plasma cortisol levels, which differentiates it from classic GCSs. This would suggest that budesonide could be the GCS of choice in the treatment of distal active UC. However, no evidence for the efficacy of oral budesonide in UC is yet available. Budenofalk $^{\circledast}$, which dissolves at $\mathrm{pH}>6.4$ and delivers budesonide in acceptable quantities to the distal colon and rectum in UC patients, may be useful for the treatment of UC patients (42). Since Entocort ${ }^{\circledR}$ can reach the transverse and descending colon (43) a comparative trial, as some authors have already suggested (6), between the two oral formulations of budesonide would be of interest. Further studies on this topic are necessary. Advances in the field of GCSs such as nitrosoglycocorticoids and selective glycocorticosteroid-receptor agonists may further improve the benefit-risk ratio (44). 


\section{ACKNOWLEDGEMENT}

Dr. Marín-Jiménez has spent six months studying inflammatory bowel disease in the Laboratory of Immunogenetics, VU University Medical Center in Amsterdam (the Netherlands), thanks to a fellowship from "Fundación para la Investigación en Gastroenterología y Hepatología", Department of Gastroenterology, Hospital General Universitario Gregorio Marañón, Madrid, Spain.

\section{REFERENCES}

1. Friend DR. Review article: issues in oral administration of locally acting glucocorticosteroids for treatment of inflammatory bowel disease. Aliment Pharmacol Ther 1998; 12: 591-603.

2. Hamedani R, Feldman RD, Feagan BG. Review article: Drug development in inflammatory bowel disease: budesonide - a model of targeted therapy. Aliment Pharmacol Ther 1997; 11 Suppl 3: 98-107; discussion 107-8.

3. Lofberg R, Danielsson A, Suhr O, Nilsson A, Schioler R, Nyberg A, et al. Oral budesonide versus prednisolone in patients with active extensive and left-sided ulcerative colitis. Gastroenterology 1996; 110: 1713-8

4. Thomsen OO, Cortot A, Jewell D, Wright JP, Winter T, Veloso FT, et al. A comparison of budesonide and mesalamine for active Crohn's disease. International Budesonide-Mesalamine Study Group. N Engl J Med 1998; 339: 370-4

5. Greenberg GR, Feagan BG, Martin F, Sutherland LR, Thomson AB, Williams CN, et al. Oral budesonide for active Crohn's disease. Canadian Inflammatory Bowel Disease Study Group. N Engl J Med 1994; 331: 836-41.

6. Fedorak RN, Bistritz L. Targeted delivery, safety, and efficacy of oral enteric-coated formulations of budesonide. Adv Drug Deliv Rev 2005; 57: 303-16

7. Lopez San Roman A, Bermejo F, Carrera E, Perez-Abad M, Boixeda D. Adherence to treatment in inflammatory bowel disease. Rev Esp Enferm Dig 2005; 97: 249-57.

8. Rodrigo L, Riestra S, Nino P, Cadahia V, Tojo R, Fuentes D, et al. A population-based study on the incidence of inflammatory bowel disease in Oviedo (Northern Spain). Rev Esp Enferm Dig 2004; 96: 296305 .

9. Gomollon F, Hinojosa J, Nos P. Budesonide and inflammatory bowel disease. Gastroenterol Hepatol 1999; 22: 525-32.

10. Klotz U, Schwab M. Topical delivery of therapeutic agents in the treatment of inflammatory bowel disease. Adv Drug Deliv Rev 2005; 57: 267-79

11. Ryrfeldt A, Edsbacker S, Pauwels R. Kinetics of the epimeric glucocorticoid budesonide. Clin Pharmacol Ther 1984; 35: 525-30.

12. Jonsson G, Astrom A, Andersson P. Budesonide is metabolized by cytochrome P450 3A (CYP3A) enzymes in human liver. Drug Metab Dispos 1995; 23: 137-42.

13. Spencer CM, McTavish D. Budesonide. A review of its pharmacological properties and therapeutic efficacy in inflammatory bowel disease. Drugs 1995; 50: 854-72.

14. McKeage K, Goa KL. Budesonide (Entocort EC Capsules): a review of its therapeutic use in the management of active Crohn's disease in adults. Drugs 2002; 62: 2263-82.

15. Edsbacker S, Andersson P, Lindberg C, Paulson J, Ryrfeldt A, Thalen A. Liver metabolism of budesonide in rat, mouse, and man. Comparative aspects. Drug Metab Dispos 1987; 15: 403-11.

16. Nyman-Pantelidis M, Nilsson A, Wagner ZG, Borga O. Pharmacokinetics and retrograde colonic spread of budesonide enemas in patients with distal ulcerative colitis. Aliment Pharmacol Ther 1994; 8: 61722.

17. Miller-Larsson A, Gustafsson B, Persson CG, Brattsand R. Gut mucosal uptake and retention characteristics contribute to the high intestinal selectivity of budesonide compared with prednisolone in the rat. Aliment Pharmacol Ther 2001; 15: 2019-25.
18. Nugent SG, Kumar D, Rampton DS, Evans DF. Intestinal luminal pH in inflammatory bowel disease: possible determinants and implications for therapy with aminosalicylates and other drugs. Gut 2001; 48 : 571-7.

19. Cui N, Friend DR, Fedorak RN. A budesonide prodrug accelerates treatment of colitis in rats. Gut 1994; 35: 1439-46.

20. Nolen H, 3rd, Fedorak RN, Friend DR. Budesonide-beta-D-glucuronide: a potential prodrug for treatment of ulcerative colitis. J Pharm Sci 1995; 84: 677-81.

21. Danielsson A, Edsbacker S, Lofberg R, Nilsson A, Nyman-Pantelidis $\mathrm{M}$, Olsson $\mathrm{O}$, et al. Pharmacokinetics of budesonide enema in patients with distal ulcerative colitis or proctitis. Aliment Pharmacol Ther 1993; 7: 401-7.

22. Lindgren S, Lofberg R, Bergholm L, Hellblom M, Carling L, Ung $\mathrm{KA}$, et al. Effect of budesonide enema on remission and relapse rate in distal ulcerative colitis and proctitis. Scand J Gastroenterol 2002; 37: 705-10.

23. Brunner M, Vogelsang H, Greinwald R, Kletter K, Kvaternik H, Schrolnberger C, et al. Colonic spread and serum pharmacokinetics of budesonide foam in patients with mildly to moderately active ulcerative colitis. Aliment Pharmacol Ther 2005; 22: 463-70.

24. Danielsson A, Lofberg R, Persson T, Salde L, Schioler R, Suhr O, et al. A steroid enema, budesonide, lacking systemic effects for the treatment of distal ulcerative colitis or proctitis. Scand J Gastroenterol 1992; 27: 9-12.

25. Hanauer SB, Robinson M, Pruitt R, Lazenby AJ, Persson T, Nilsson LG, et al. Budesonide enema for the treatment of active, distal ulcerative colitis and proctitis: a dose-ranging study. U.S. Budesonide enema study group. Gastroenterology 1998; 115: 525-32.

26. Bar-Meir S, Fidder HH, Faszczyk M, Bianchi Porro G, Sturniolo GC, Mickisch O, et al. Budesonide foam vs. hydrocortisone acetate foam in the treatment of active ulcerative proctosigmoiditis. Dis Colon Rectum 2003; 46: 929-36.

27. Hammond A, Andus T, Gierend M, Ecker KW, Scholmerich J, Herfarth $\mathrm{H}$. Controlled, open, randomized multicenter trial comparing the effects of treatment on quality of life, safety and efficacy of budesonide foam and betamethasone enemas in patients with active distal ulcerative colitis. Hepatogastroenterology 2004; 51: 1345-9.

28. Marshall JK, Irvine EJ. Rectal corticosteroids versus alternative treatments in ulcerative colitis: a meta-analysis. Gut 1997; 40: 775-81.

29. Nos P, Hinojosa J, Gomollon F, Ponce J. Budesonide in inflammatory bowel disease: a meta-analysis. Med Clin (Barc) 2001; 116: 47-53.

30. Danielsson A, Hellers G, Lyrenas E, Lofberg R, Nilsson A, Olsson O, et al. A controlled randomized trial of budesonide versus prednisolone retention enemas in active distal ulcerative colitis. Scand J Gastroenterol 1987; 22: 987-92.

31. Group DBS. Budesonide enema in distal ulcerative colitis. A randomized dose-response trial with prednisolone enema as positive control. The Danish Budesonide Study Group. Scand J Gastroenterol 1991; 26: 1225-30.

32. Bianchi Porro G, Prantera C, Campieri M, Petrillo M, Campanini M, Gionchetti $\mathrm{P}$, et al. Comparative trial of methylprednisolone and budesonide enemas in active distal ulcerative colitis. Eur J Gastroenterol Hepatol 1994; 6: 125-30.

33. Ostergaard-Thomsen O, Andersen T, Langholz E, Lofberg R, Malchow-Moller A, Matzen P, et al. Lack of adrenal gland suppression with budesonide enema in active distal ulcerative colitis. Eur J Gastroenterol Hepatol 1994; 6: 507-11.

34. Lofberg R, Ostergaard Thomsen O, Langholz E, Schioler R, Danielsson A, Suhr O, et al. Budesonide versus prednisolone retention enemas in active distal ulcerative colitis. Aliment Pharmacol Ther 1994; 8: 623-9.

35. Tarpila S, Turunen U, Seppala K, Aukee S, Pikkarainen P, Elomaa I, et al. Budesonide enema in active haemorrhagic proctitis - a controlled trial against hydrocortisone foam enema. Aliment Pharmacol Ther 1994; 8: 591-5.

36. Bayless T, Sninsky C, Group ftUBES. Budesonide enema is an effective alternative to hydrocortisone enema in active distal ulcerative colitis. In: Gastroenterology; 1995; 1995. p. A778.

37. Lemann M, Galian A, Rutgeerts P, Van Heuverzwijn R, Cortot A Viteau JM, et al. Comparison of budesonide and 5-aminosalicylic acid enemas in active distal ulcerative colitis. Aliment Pharmacol Ther 1995; 9: 557-62. 
38. Rufle W, Fruhmorgen P, Huber W, Kimmig JM. Budesonide foam as a new therapeutic principle in distal ulcerative colitis in comparison with mesalazine enema. An open, controlled, randomized and prospective multicenter pilot study. Z Gastroenterol 2000; 38: 28793.

39. Lamers C, Meijer J, Engels L, Bos L, van Hogezand R, Driessen W, et al. Comparative study of the topically acting glucocorticosteroid budesonide and 5-aminosalicylic acid enema therapy of proctitis and proctosigmoiditis. Gastroenterology 1991; 100: A223.

40. Sambuelli A, Boerr L, Negreira S, Gil A, Camartino G, Huernos S, et al. Budesonide enema in pouchitis - a double-blind, double-dummy, controlled trial. Aliment Pharmacol Ther 2002; 16: 27-34.
41. Keller R, Stoll R, Foerster EC, Gutsche N, Domschke W. Oral budesonide therapy for steroid-dependent ulcerative colitis: a pilot trial. Aliment Pharmacol Ther 1997; 11: 1047-52.

42. Kolkman JJ, Mollmann HW, Mollmann AC, Pena AS, Greinwald R, Tauschel HD, et al. Evaluation of oral budesonide in the treatment of active distal ulcerative colitis. Drugs Today (Barc) 2004; 40: 589-601.

43. Edsbacker S, Bengtsson B, Larsson P, Lundin P, Nilsson A, Ulmius J, et al. A pharmacoscintigraphic evaluation of oral budesonide given as controlled-release (Entocort) capsules. Aliment Pharmacol Ther 2003; 17: 525-36.

44. Buttgereit F, Burmester GR, Lipworth BJ. Optimised glucocorticoid therapy: the sharpening of an old spear. Lancet 2005; 365: 801-3. 